\title{
TRANSFUSÃO MACIÇA: Uma abordagem didática para graduandos
}

\author{
MASSIVE TRANSFUSION: \\ A review article for medical students

\begin{abstract}
Luiz PC Faggioni ${ }^{1}$; Dimas Tadeu Covas ${ }^{2}$; Eduardo Mendel Balbi Filho ${ }^{3}$; Marcos Antonio de Pádua ${ }^{3}$;
\end{abstract} \\ Raphael A. Tobias"; Maurice J. De Rosa ${ }^{4}$ \& Fábio Papa ${ }^{3}$
}

\begin{abstract}
${ }^{1}$ Mestrando em Hematologia e Hemoterapia. ${ }^{2}$ Livre Docente do Departamento de Clínica Médica. ${ }^{3}$ Acadêmicos de Medicina. ${ }^{4}$ Residentes do Departamento de Cirurgia e Anestesiologia. Faculdade de Medicina de Ribeirão Preto da Universidade de São Paulo. CorRespondÊncia: Luiz Paulo Cicogna Faggioni - Hemocentro de Ribeirão Preto - Rua Tenente Catão Roxo, 2501 - Ribeirão Preto, São Paulo, Brasil - CEP: 14051-140 - E-mail: faggioni@pegasus.fmrp.usp.br
\end{abstract}

FAGGIONI LPC; COVAS DT; BALBI FILHO EM; PÁDUA MA; TOBIAS RA; DE ROSA MJ \& PAPA F. Transfusão maciça: uma abordagem didática para graduandos. Medicina, Ribeirão Preto, 32: 438-443, out./dez. 1999.

RESUMO: O texto aborda, sucintamente, a fisiopatologia e as complicações de transfusões maciças em pacientes politraumatizados, mostrando a correlação entre a apresentação clínica inicial e perdas sangüíneas estimadas, assim como a terapêutica transfusional racional a ser utilizada.

UNITERMOS: Traumatismo Múltiplo. Transfusão de Sangue. Hemorragia. Choque Hemorrágico.

\section{INTRODUÇÃO}

Sangramentos agudos de grande volume têm sido sempre um desafio aos serviços de emergência.

A necessidade de compreender a fisiopatologia do choque hemorrágico e a adoção de uma terapêutica adequada remontam à I Guerra Mundial, embora progressos importantes só tenham sido feitos a partir da utilização de plasma como expansor de volume, na II Grande Guerra.

Na década de 50, o conflito na Coréia, e, nos anos 60 e 70, a Guerra do Vietnã, trouxeram aos cirurgiões americanos maiores conhecimentos e experiência sobre choques hemorrágicos.

Recentemente, a necessidade de grande suporte hemoterápico aos transplantes hepáticos levou ao desenvolvimento de protocolos ainda mais racionais para utilização monitorada, pelo laboratório de hematologia, de hemocomponentes.

$\mathrm{O}$ entendimento de haver, no choque por per- das maciças de sangue, o comprometimento tanto da perfusão tecidual como da oxigenação é crítico, assim como as complicações decorrentes do quadro de base (choque e hipóxia tecidual) e secundárias à terapêutica.

Quando estamos diante de um sangramento maciço em uma sala de emergência ou em um centro cirúrgico? Como diagnosticar o quadro, como evolui e qual a conduta mais adequada no tratamento do sangramento maciço?

\section{DEFINIÇÕES}

A definição habitualmente utilizada para transfusão maciça é: a reposição de, pelo menos, uma volemia, em um intervalo de até 24 horas.

Outras definições foram utilizadas, como:

- reposição de $50 \%$ da volemia em três horas;

- transfusão de mais de 20 unidades de concentrado de hemácias. 
Esses critérios abordavam um diagnóstico retrospectivo de um sangramento maciço. Com o objetivo de abordar rapidamente este tipo de paciente, outras definições se fizeram necessárias.

Atualmente, os serviços de hemoterapia têm adotado uma visão bem dinâmica: prevêem, antecipadamente, considerando o estado clínico apresentado pelos pacientes, a necessidade de quatro ou mais unidades de concentrado de hemácias.

Para o atendimento mais completo das transfusões maciças, é necessária uma revisão dos conceitos de volume/perfusão e oxigenação tecidual, bem como a diferença que há entre os conceitos.

\section{HIPOVOLEMIA E HIPOXEMIA}

Trabalhos clínicos experimentais têm mostrado que perdas de até $75 \%$ de massa eritrocitária podem ser toleradas, desde que seja mantida a volemia. Porém, perdas volêmicas de cerca de $30 \%$ são freqüentemente fatais.

Esses fatos podem ser analisados, quando calculamos a diferença entre o nosso suprimento de oxigênio e o nosso consumo.

$\mathrm{O}$ suprimento de $\mathrm{O}_{2}$ pode ser avaliado pela expressão :

Suprimento de $\mathrm{O}_{2}=$ débito cardíaco $x$ conteúdo de $\mathrm{O}_{2}$ arterial

Suprimento de $\mathrm{O}_{2}=5$ litros / minuto $\times 200 \mathrm{~mL}$ de $\mathrm{O}_{2} /$ litro

Suprimento de $\mathrm{O}_{2}=1000 \mathrm{~mL} \mathrm{O}_{2} /$ minuto

* Em um adulto em repouso, com função cardíaca e hemoglobina em níveis fisiológicos

Nosso consumo de oxigênio pode ser calculado pela diferença arterial e venosa:

Consumo de $\mathrm{O}_{2}=$ débito cardíaco $x$ (conteúdo arterial - venoso)

Consumo de $\mathrm{O}_{2}=5$ litros/minuto $\times\left(200 \mathrm{~mL} \mathrm{O}_{2} /\right.$ litro $\left.-150 \mathrm{~mL} \mathrm{O}_{2} \mathrm{~L}\right)$

Consumo de $\mathrm{O}_{2}=250 \mathrm{~mL} \mathrm{O}_{2} / \mathrm{min}$

Suprimento de $\mathrm{O}_{2}\left(1000 \mathrm{~mL} \mathrm{O}_{2} /\right.$ minuto $)$ - Consumo de $\mathrm{O}_{2}$ $(250 \mathrm{~mL} \mathrm{O} / \mathrm{min})=750 \mathrm{mLO}_{2} /$ minuto

Portanto, utilizamos somente $1 / 4$ do nosso suprimento de $\mathrm{O}_{2}$ em situações fisiológicas. Embora nossa reserva seja grande, pacientes com hemorragias estão sob stress, o que aumenta o consumo de $\mathrm{O}_{2}$.

Sendo assim, a atenção inicial, em pacientes com hemorragias, deve ser dada à manutenção da volemia (reexpansão volumétrica) e, posteriormente, deveremos nos ater à capacidade de transporte de oxigênio.
O trabalho de Lundsgaard-Hansen ${ }^{(1)}$ reforça essa seqüência, conforme será visto posteriormente.

Os expansores plasmáticos têm sido fonte de importantes discussões e estudos clínicos, e eles se dividem em cristalóides e colóides.

Cristalóides, como solução de Ringer e salina $0,9 \%$, têm a vantagem do menor custo, facilidade de manuseio, a segurança, a facilidade da remoção no caso de sobrecarga, mas, por outro lado, apresentam risco de diminuição da pressão oncótica do compartimento intravascular.

Os colóides, solução de albumina a $20 \%$, Hidroxietilestarch e Dextran, têm um custo maior e não podem ser removidos em caso de sobrecarga.

Há, atualmente, uma forte tendência para que a reexpansão inicial de pacientes em choque hemorrágico seja feita com cristalóides, devido às vantagens citadas.

E, agora, como diagnosticar as perdas volumétricas iniciais? É possível associar a apresentação clínica inicial de um paciente com graus diferentes de perdas de volume, no sangramento maciço?

Qual a conduta inicial, levando em conta tanto a hipovolemia quanto a hipóxia, devido à perda de capacidade de transporte de $\mathrm{O}_{2}$ ?

\section{AVALIAÇÃO CLÍNICA INICIAL}

O Clinical practice of transfusion medicine ${ }^{(2)}$ apresentou uma tabela de fácil entendimento que correlaciona perdas sangüíneas estimadas e apresentação clínica inicial de pacientes (Tabela I).

Se o quadro inicial, apesar das medidas de medidas adequadas, não for estabilizado, quais seriam os próximos passos?

Lundsgaard-Hansen trabalharam com modelos experimentais de perdas progressivas de sangue.

\section{MODELO DE LUNDSGAARD-HANSEN E TERAPIA TRANSFUSIONAL}

\subsection{Modelo de Lundsgaard-Hansen - Como in- terpretar a curva?}

Lundsgaard \& Hansen apresentaram uma curva (Figura 1) que prediz os pontos críticos de cada componente sangüíneo, correlacionando-os com a evolução da perda sangüínea.

No eixo das abcissas, temos a porcentagem de perdas sangüíneas estimadas e, no eixo das ordenadas, os pontos críticos de cada componente do sangue. 
Tabela I - Perda volêmica estimada, baseada na apresentação clínica inicial

\begin{tabular}{|c|c|c|c|c|}
\hline & Classe I & Classe II & Classe III & Classe IV \\
\hline $\begin{array}{l}\text { Perda sangüínea } \\
\text { (estimada em mL) }\end{array}$ & até 750 & $750-1500$ & $1500-2000$ & 2000 \\
\hline $\begin{array}{l}\text { Perda sangüínea } \\
\text { (estimada em \%) }\end{array}$ & até 15 & de $15-30$ & de $30-40$ & $>40$ \\
\hline Freqüência cardíaca/pulso & $<100$ & $>100$ & $>120$ & $>140$ \\
\hline Pressão & Normal & Normal & Diminuída & Diminuída \\
\hline Pressão/Pulso $(\mathrm{mmHg})^{*}$ & $\begin{array}{l}\text { Normal ou } \\
\text { elevada }\end{array}$ & Diminuída & Diminuída & Diminuída \\
\hline $\begin{array}{l}\text { Freqüência Respiratória } \\
\text { (incursões/minuto) }\end{array}$ & $14-20$ & $20-30$ & $30-40$ & $>35$ \\
\hline Diurese (mL/hora) & $>30$ & $20-30$ & $5-15$ & Desprezível \\
\hline Estado de consciência & $\begin{array}{l}\text { Discreta } \\
\text { ansiedade }\end{array}$ & $\begin{array}{l}\text { Moderada } \\
\text { ansiedade }\end{array}$ & Ansioso e confuso & $\begin{array}{l}\text { Confuso e } \\
\text { letárgico }\end{array}$ \\
\hline Fluido terapia** & Cristalóide & Cristalóide & $\begin{array}{l}\text { Cristalóide e } \\
\text { sangue }^{\star \star \star}\end{array}$ & $\begin{array}{l}\text { Cristalóide e } \\
\text { sangue }^{\star * *}\end{array}$ \\
\hline \multicolumn{5}{|c|}{$\begin{array}{l}\text { * Pressão de pulso = diferença entre pressão sistólica e diastólica } \\
\text { ** É importante lembrarmos que apenas } 1 / 3 \text { do volume de cristalóide infundido permanece no intravascular, portanto as perdas } \\
\text { sangüíneas, quando repostas com cristalóide devem manter a proporção de três volumes de cristalóide para cada volume de sangue } \\
\text { perdido. Exemplo: pacientes que se enquadram na classe I devem receber cerca de } 2000 \mathrm{~mL} \text { de cristalóide para manter a volemia. } \\
\text { ***Por sangue entenda-se concentrado de hemácias, quatro unidades. }\end{array}$} \\
\hline
\end{tabular}

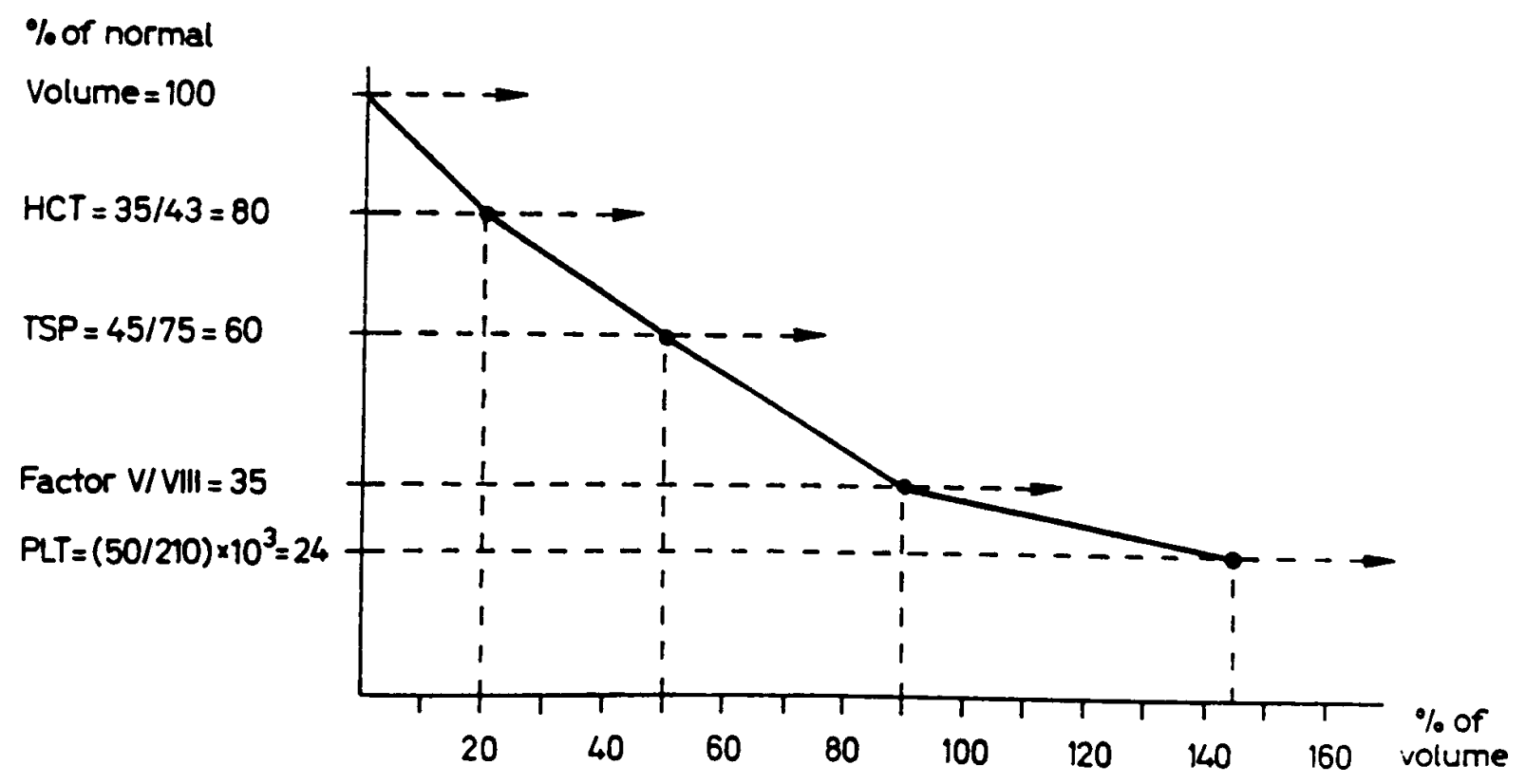

Figura 1 - Modelo de Lundsgaard - Hansen. 
O ponto crítico do volume é de 100 a $80 \%$, confirmando os trabalhos experimentais.

Em seguida, há comprometimento da oxigenação em perdas que variam de 20 a $50 \%$.

Entre 50 e $90 \%$, há comprometimento da porcentagem de proteína sérica total (representado no gráfico por TSP), comprometendo o equilíbrio oncótico.

Em perdas de 90 a $145 \%$, os níveis de fatores mais lábeis, e, também, de maior peso molecular, de coagulação (fatores V e VIII) estão comprometidos.

Perdas superiores a $150 \%$ já apresentam trombocitopenia dilucional.

Isso é o que corresponde à maioria das situações clínicas, contudo, apesar de a ordem estar correta, os níveis críticos podem variar e serem atingidos posteriormente ao predito pelo modelo.

De acordo com a curva, podemos extrapolar uma abordagem racional do uso de hemocomponentes e hemoderivados.

\subsection{Terapêutica Transfusional (como utilizar a curva)}

- Sangramento de menos de $20 \%$ da volemia: somente o volume deve ser reposto, com o uso de cristalóide, se a $\mathrm{Hb}$ inicial for maior que $10-11 \mathrm{~g} / \mathrm{dL}$.

- Sangramento entre 20 e $50 \%$ da volemia: reexpandir volume (cristalóide) e transfundir concentrado de hemácias, se o quadro clínico sugerir sangramento próximo de $50 \%$ do volume sangüíneo.

- Sangramento entre 50 e $100 \%$ da volemia: reexpandir volume, concentrado de hemácias e elevar a pressão coloidosmótica (albumina 5\% é a alternativa mais segura e fisiológica).

- Sangramento maior que $100 \%$ da volemia: reexpandir volume, concentrado de hemácias, elevar a pressão coloidosmótica, crioprecipitado (que contém fatores de alto peso molecular da coagulação fatores $\mathrm{V}$ e VIII) e concentrado de plaquetas. As doses terapêuticas de crioprecipitado dependem de avaliação laboratorial TTPa maior que $25 \%$, ou, na ausência do laboratório, uma unidade para cada $10 \mathrm{Kg}$ de peso do paciente, de acordo com a evolução das perdas. No concentrado de plaquetas, deve ser utilizada uma unidade para cada $7 \mathrm{Kg}$ do paciente.

\section{COMPLICAÇÕES}

\subsection{O 2,3 difosfoglicerato (2,3 DPG), a lesão de estoque e a importância tranfusional}

A transfusão de concentrado de hemácias, ou papa de hemácias, tem como função restabelecer a capacidade de transporte de oxigênio, evitando hipoxemia tecidual.

Uma questão surge em tal caso: o armazenamento ou a adição de conservantes aos concentrados de hemácias alteram a capacidade de transporte ou liberação de $\mathrm{O}_{2}$ das hemácias acondicionadas?

\section{Bioquímica}

O transporte de oxigênio, feito pela hemoglobina $(\mathrm{Hb})$, pode ser resumido em nível tecidual através do seguinte equilíbrio:

$$
\mathrm{Hb}+\mathrm{O}_{2} \leftrightarrow \mathrm{HbO}_{2}
$$

Em tecidos onde é baixa a tensão de oxigênio, o equilíbrio é deslocado para a esquerda, liberando maior quantidade de $\mathrm{O}_{2} \mathrm{Na}$ circulação pulmonar, onde a tensão de $\mathrm{O}_{2}$ é alta, há deslocamento do equilíbrio para a direita.

\section{O 2,3 DPG}

O 2,3 difosfoglicerato ou bifosfoglicerato (Figura 2) é uma pequena molécula com alta carga negativa, que se combina não covalentemente à cavidade central de uma molécula de hemoglobina desoxigenada, diminuindo, assim, a afinidade desta pelo oxigênio, como mostra a Figura 3. Portanto, desloca o equilíbrio para a esquerda, facilitando a liberação de $\mathrm{O}_{2}$ em tecidos onde há baixa tensão do mesmo.

Nos tecidos com alta tensão de $\mathrm{O}_{2}$, a molécula de 2,3 DPG é deslocada do centro da hemoglobina desoxigenada, facilitando a captação de $\mathrm{O}_{2}$.

\section{A lesão de estoque}

Devemos entender por lesão de estoque qualquer alteração bioquímica que ocorra em hemocomponentes, durante o processamento e estoque.

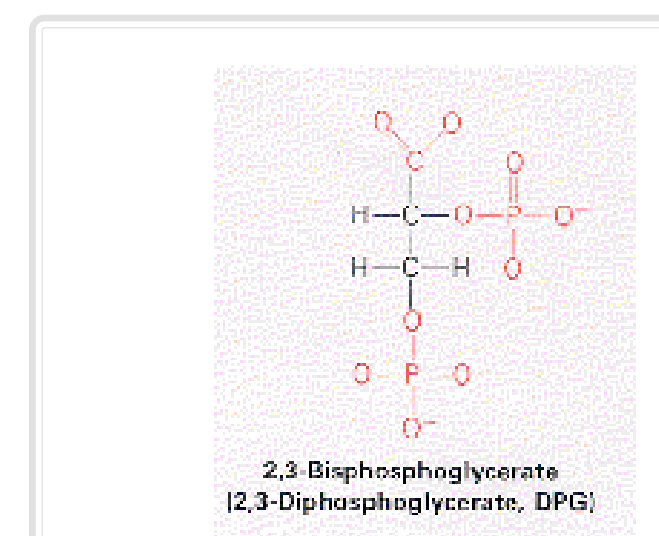

Figura 2 - Desoxihemoglobina e o sítio de ligação do 2,3 DPG. 


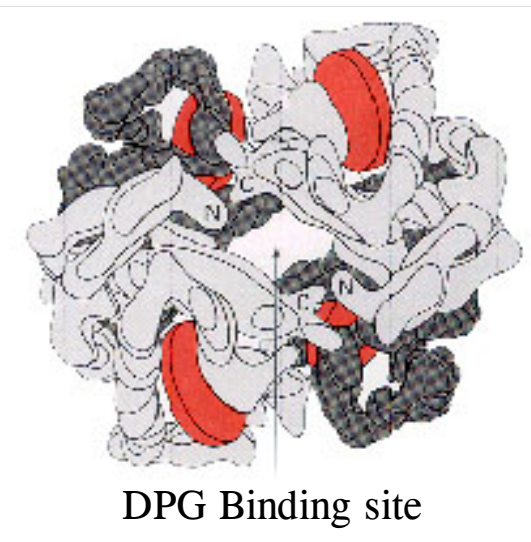

Figura 3 - Estrutura química do 2,3 DPG.

A concentração de 2,3 DPG cai gradualmente durante o estoque do concentrado de hemácias até níveis próximos de zero. A queda na concentração de 2,3 DPG é dependente do tipo de conservante. O conservante mais comumente utilizado é o CPDA 1 , que mantém níveis adequados de 2,3 DPG até cinco dias pós estoque.

Por esse motivo, em transfusões maciças, como, por exemplo, exsangüineotransfusões, fazse necessário o uso de concentrado de hemácias com tempo de estoque menor que três dias.

A normalização dos níveis de 2,3 DPG ocorre cerca de 12 a 24 horas após a transfusão.

\subsection{O equilíbrio acidobásico}

É importante lembrarmos que, em todo paciente com hemorragia severa ou choque, há algum grau de acidose metabólica. Entretanto, alguns pontos devemos ter em vista.

Muitos estudos têm mostrado que o distúrbio acidobásico mais comum em pacientes que recebem transfusões maciças é a alcalose metabólica. Apesar de o choque circulatório ocasionar certa acidose metabólica, temos de lembrar que o conservante do concentrado de hemácias é o citrato, um quelante do cálcio.

In vivo, após a passagem hepática, o citrato é transformado em bicarbonato, que, em circulação, leva à alcalose. Devido aos efeitos provenientes da alcalose (hipocalcemia, hipocalemia e desvio à esquerda na curva de dissociação da hemoglobina), devemos evitar a correção da acidose inicial de pacientes chocados que recebem tranfusões maciças, pois podemos, mais tarde, potencializar a alcalose, que é uma entidade clínica de difícil tratamento.

\subsection{Os efeitos do citrato}

Em pacientes com perdas sangüíneas importantes, e conseqüente choque circulatório, há uma diminuição da perfusão hepática. Assim, ocorre um aumento na concentração plasmática do citrato, devido à queda no seu metabolismo. Sendo o citrato, um quelante de cálcio, pode ocorrer hipocalcemia, ocasionando arritmias cardíacas.

Quando se fizer necessária a reposição de cálcio, é importante lembrarmos que não se deve administrar cálcio pela mesma via de administração do sangue, pois pode ocorrer formação de microêmbolos (isto ocorre pela anulação do efeito quelante do citrato).

\subsection{A Hipotermia}

O concentrado de hemácias é armazenado entre 1 e $6{ }^{\circ} \mathrm{C}$. Em pequenas transfusões, essa temperatura não tem repercussões importantes. Mas, quando se trata de transfusões maciças em pacientes chocados, hipotermia é um problema para o qual devemos ter atenção.

A hipotermia pode causar arritmias ventriculares, que podem, ainda, ser potencializadas pela hipocalcemia (como discutido acima), levando a um pobre desempenho ventricular esquerdo. Essas arritmias ocorrem com freqüência, principalmente quando a administração do concentrado de hemácias é feita por um cateter central, posicionado próximo ao sistema de condução cardíaco.

Há várias maneiras de se contornarem esses efeitos, como, por exemplo, afastar o cateter central do átrio, diminuir a taxa de infusão, ou, ainda, utilizar aquecedores de sangue, que não devem elevar a temperatura do sangue acima de $42^{\circ} \mathrm{C}$, para evitar hemólise térmica do concentrado.

Outro mecanismo que podemos utilizar é a adição de salina previamente aquecida junto ao concentrado de hemácias antes da infusão.

\subsection{Distúrbios de coagulação}

Tais distúrbios são decorrentes do quadro de base ou complicação da terapêutica.

O traumatismo múltiplo de órgãos pode levar à coagulação intravascular disseminada (CIVD) devido à liberação de fatores ativadores da cascata da coagulação; o mesmo ocorre devido à acidose, hipotermia (decorrente do choque ou devido à infusão rápida de hemocomponentes que são estocados à temperatura de 1 a $6^{\circ} \mathrm{C}$, como concentrados de hemácias) e hipóxia prolongadas.

A rápida e maciça infusão de cristalóides e 
concentrados de hemácias, na abordagem inicial do paciente, se não levarmos em conta o ponto crítico de fatores lábeis da coagulação, e estes não forem repostos a seu tempo certo, pode acarretar o que é conhecido como coagulopatia diluicional, a diluição dos fatores de coagulação e conseqüente distúrbio associado, piora ou dificuldade de estancar sangramentos.

Esses quadros quase sempre se sobrepõem. O alargamento tanto do Tempo de Protrombina quanto do Tempo de Tromboplastina Parcial ativado faz o diagnóstico laboratorial desses quadros. A presença de dímeros de fibrina é positiva na coagulação intravascular disseminada e negativa na coagulopatia diluicional.

\section{CONCLUSÃO}

A abordagem terapêutica e programada de pacientes politraumatizados, com sangramentos maciços, tem se desenvolvido juntamente com o entendimento da fisiopatologia de choques hemorrágicos, bem como com o conhecimento das complicações decorrentes do quadro de base e secundárias à terapêutica. Tais conhecimentos têm mudado, de forma significativa, tanto a morbidade quanto a mortalidade desses pacientes, também permitindo o maior sucesso de cirurgias, quando é necessário um grande suporte de hemoderivados e hemocomponentes.

FAGGIONI LPC; COVAS DT; BALBI FILHO EM; PÁDUA MA; TOBIAS RA; DE ROSA MJ \& PAPA F. Massive transfusion: a review article for medical students. Medicina, Ribeirão Preto, 32: 438-443, oct./dec. 1999.

ABSTRACT: This review article for medical students approaches the basis and complications of massive transfusion in trauma patients, correlating clinical aspects with estimated blood losses.

UNITERMS: Multiple Trauma. Blood Transfusion. Hemorrhage. Schock, Hemorrhagic.

\section{BIBLIOGRAFIA CONSULTADA}

1 - AMERICAN ASSOCIATION OF BLOOD BANKS. Technical manual. 12th ed. AABB, Bethesda, 1996.

2 - FARKAS DH. Clinics in laboratory medicine: Blood banking. W.B. Saunders, Philadelphia, 1996.

3 - STRYER LT. Biochemistry. 3th ed. Stanford University, Stanford. 1988.

4 - SUMMERS SH. Transfusion therapy: guidelines for practice. AABB, Bethesda, 1990.

\section{REFERÊNCIAS BIBLIOGRÁFICAS}

1 - LUNDSGAARD-HANSEN P. Component therapy of surgical haemorrhage. Bibl Hematol 46: 147-169, 1980.

2 - ROSS S \& JETER E. Emergency surgery - trauma and massive transfusion. In: PETZ LD. Clinical practice of transfusion medicine. 3 th. ed. Churchill Livingstone, New York, chap. 25, p. 565, 1996.

Recebido para publicação em 08/12/1999

Aprovado para publicação em 19/01/2000 